\title{
A Novel Layered Bidirectional Equalizer Based on a Buck-Boost Converter for Series-Connected Battery Strings
}

\author{
Shubiao Wang ${ }^{1,3}$, Longyun Kang ${ }^{1,3, *}$, Xiangwei Guo ${ }^{2}$, Zefeng Wang ${ }^{1,3}$ and Ming Liu ${ }^{1,3}$ \\ 1 New Energy Research Center, School of Electric Power, South China University of Technology, \\ Guangzhou 510640, China; epwangshubiao@mail.scut.edu.cn (S.W.); epzfwang@mail.scut.edu.cn (Z.W.); \\ lium@scut.edu.cn (M.L.) \\ 2 College of Electrical Engineering and Automation, Henan Polytechnic University, Jiaozuo 454000, China; \\ gxw@hpu.edu.cn \\ 3 Guangdong Key Laboratory of Clean Energy Technology, School of Electric Power, \\ South China University of Technology, Guangzhou 510640, China \\ * Correspondence: lykang@scut.edu.cn; Tel.: +86-137-2809-8863
}

Received: 13 June 2017; Accepted: 11 July 2017; Published: 17 July 2017

\begin{abstract}
To eliminate the influence of the inconsistency on the cycle life and the available capacity of battery packs, and improve the balancing speed, a novel inductor-based layered bidirectional equalizer (IBLBE) is proposed. The equalizer is composed of two layers of balancing circuits connected in parallel. Each layer contains multiple balancing sub-circuits based on buck-boost converters. These balancing sub-circuits can equalize the corresponding cells simultaneously, and allow the dynamic adjustment of equalization path and equalization threshold. Analysis and simulation results demonstrate the IBLBE has a higher level balancing speed than other equalizers based on switched-capacitor or switched-inductor converters, and reduces the balancing time by $30 \%$ compared to existing inductor-based parallel architecture equalizers (PAEs). Experimental results are presented to validate the analysis and effectiveness of the proposed equalizer.
\end{abstract}

Keywords: battery string; layered bidirectional equalizer; buck-boost converter; balancing speed

\section{Introduction}

Since individual battery cells have limited voltage and capacity, power batteries require a variety of series or parallel combinations to achieve the voltage or capacity level for various applications [1,2]. However, due to manufacturing inconsistencies, and differences in the operating temperature and unique performance characteristics of each single cell, the cells connected in series may suffer from a serious imbalance between cell voltages or state of charge (SOCs) after many charging and discharging cycles [3]. The imbalance of SOC can cause some of the cells to become overcharged or over discharged. This reduces the usable capacity of battery strings, shortens the lifetime, and even poses a safety hazard (e.g., an explosion or fire, etc.). Equalization for battery strings could be realized to prevent these phenomena and prolong battery string lifetimes.

Numerous equalization topologies and balancing methods have been proposed [4-25], and they are well summarized in [5-7]. The equalization topologies are usually categorized as either passive or active circuits.

Passive equalization is the most straightforward and cheapest method. However, the excess energy is converted into heat rather than stored, which leads to energy waste and thermal management problems [5,7].

The active equalization circuits transfer energy to energy storage elements, such as capacitors, inductors and transformers. Thus, these active equalization circuits can generally be categorized as 
capacitor-based [8-14], inductor-based [15-23] or transformer-based [24-26] circuits, each of which has its own advantages and disadvantages in terms of speed, accuracy, cost, size and efficiency.

With the advantages of small size, low cost, and easy control, switched-capacitor (SC) power conversion circuits are successfully used to equalize the cell voltages of series-connected battery strings [8]. Resonant equalizers are also developed based on LC (inductor and capacitor) quasi-resonant circuits in [12-14]. The equalizers based on LC quasi-resonant circuits can realize zero-current switching and reduce the switching loss of switches. However, the balancing speed of these circuits becomes lower when the cell voltage difference is small $[6,8,13]$. Shang and Li have done some work to improve the balancing speed of LC quasi-resonant equalizers by using buck-boost converters to enlarge the voltage difference across the LC quasi-resonant converter, but it compromises on converters efficiency $[6,13]$. Transformer-based equalization circuits have a very high balancing speed and high level of integration, but they need a transformer with multiple outputs for all the cells. This transformer is expensive and difficult to design for a large number of cells [3]. In addition, the transformer-based equalizers have poor expandability and large transformer magnetic flux leakage $[7,15]$. Thus, the transformer-based equalizers have difficulties to use in a large battery string. Inductor-based equalization circuits include buck-boost, Cûk, Sepic converters and so on. The equalization circuits proposed in [15-22] belong to this type. Inductor-based equalization circuits can realize bidirectional energy flow with higher balancing efficiency, but they often require a complex switch array and a precise control algorithm $[8,24]$.

The topologies discussed above can solve the imbalance problem quickly if there are only a few cells in series. However, many of these equalizers suffer from long balancing times for a large number of battery cells. One reason why the balancing process is slow is that the equalizers are connected in series and the balancing speed is thus limited to the capacity of a single equalizer on the equalization path $[3,15]$. The balancing speed is not only related to the equalization topology, but also to the number of equalization path operating in parallel.

Parallel structures proposed in $[9,10,15,16,24,25]$ have a faster equalization speed, but the transformer-based parallel structures $[25,26]$ have design difficulties for a large number of cells. To expand the equalization path, Han has proposed a parallel architecture equalizer (PAE) based on buck-boost converters for battery strings in [15], as shown in Figure 1. In the PAE, all the balancing sub-circuits are placed in parallel layers. All the layers can equalize their corresponding cell groups simultaneously. Han has done a systematic comparison between parallel architecture equalizer (PAE) based on buck-boost converters, traditional inductor-based adjacent equalizer (IBAE) [18], parallel architecture equalizer based on a multi-wind transformer (PAEBMWT) [25] and double-tiered switched-capacitor equalizer (DTSCE) [10]. The comparison results show that the PAE has a good performance in balancing speed, balancing efficiency, and control complexity. The balancing time of PAE is the shortest, and it can be reduced by $50 \%$, compared with IBAE. In addition to PAE, some parallel architecture equalizers realized by switched-capacitor converters $[8,9]$ and switched-inductors $[16,20]$ will be introduced in detail in Section 4.

In consideration of the advantages and disadvantages of the equalization methods discussed above, a novel inductor-based layered bidirectional equalizer (IBLBE) with the advantages of series-parallel connected balancing path and high level balancing speed is proposed. The main idea of the IBLBE is modularizing the battery string with layered balancing circuits based on buck-boost converters to realize fast active equalization in different layers synchronously, especially for large battery strings. The IBLBE has a higher level balancing speed than other equalizers based on switched-capacitor or switched-inductor converters.

In this paper, the IBLBE is proposed and analyzed. Its circuit structure and equalization principle are introduced in Section 2. The models and calculation of the key parameters are derived in Section 3. In Section 4, the simulation results and comparison with several existing equalizer are presented. The experimental result is shown in Section 5, followed by the conclusion in Section 6 . 


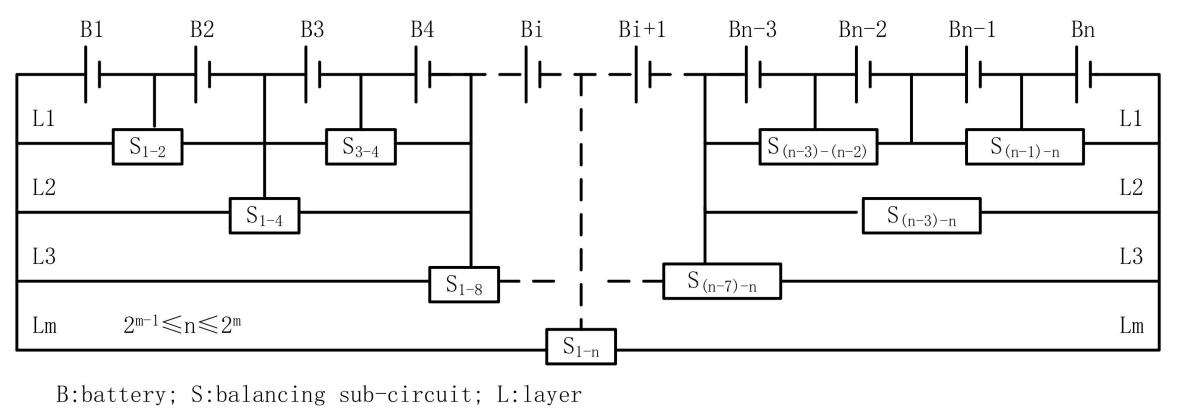

Figure 1. The parallel architecture equalizer proposed in [15].

\section{Proposed Equalizer Scheme}

\subsection{Structure of the Proposed Equalizer}

Figure 2 shows the system configuration of the proposed equalizer. Figure $2 \mathrm{a}$ is the structure of the equalizer; Figure $2 b$ is the schematic diagram of the bottom balancing circuit; Figure $2 c$ is the schematic diagram of the balancing sub-circuit.

The battery string connected in series is subdivided into $N$ modules, and each module contains $n$ individual cells. The $N$ battery modules are divided into two parts with cutoff point $K$. If $N$ is an even number, $N=2 \times K$; if $N$ is an odd number, $N=2 \times K-1$. Every battery module $M_{i}$ is equipped with a balancing sub-circuit $E_{i}$, which controls the charge transfer between $M_{i}$ and other modules. Each battery module is divided into two parts with cutoff point $k$. If $n$ is an even number, $n=2 \times k$; if $n$ is an odd number, $n=2 \times k-1$. Every individual cell is equipped with a balancing sub-circuit $S_{i}$, which controls the charge transfer between $B_{i}$ and other individual cells in this module.

The balancing sub-circuits $E_{i}$ and $S_{i}$ are implemented by buck-boost converters, which allow bidirectional energy flows. The balancing sub-circuits $E_{i}$ compose the upper balancing circuits, which are used to achieve the equalization between battery modules. The balancing sub-circuits Si compose the bottom balancing circuits which are used for equalization between cells in each module. The upper layer circuits and the bottom layer circuit are connected in parallel and can be operated simultaneously, which extends the balancing path and can achieve fast equalization for a large series connected battery string.

The proposed equalizer IBLBE can achieve the dynamic adjustment of equalization path and equalization threshold, described as follows:

- Dynamic adjustment of the equalization path

The balancing sub-circuit $E_{i}$ provides equalization path for module $M_{i}$, and the balancing sub-circuit $S_{i}$ provides equalization path for individual cell $B_{i}$. Each balancing sub-circuit can be operated independently according to the specific algorithm, which is determined according to different requirements. For instance, the equalizer operates $E_{i}(s)$ in upper layer and $S_{j}(s)$ in bottom layer concurrently to realize fast active equalization for a large battery string. Furthermore, the $S_{r}$ and $S_{t}$ (suppose $B_{r}$ and $B_{t}$ are in the same module, $V_{B_{r}}>V_{B_{j}}>V_{B_{t}}, r, j, t=1,2, \ldots, n$ ) can be operated synchronously to balance the most overcharged cell $B r$ and most undercharged cell $B t$ at the same time.

- Dynamic adjustment of equalization threshold

The equalization circuits have two equalization thresholds; one for the battery modules and the other for the individual cells in the same module. If the two thresholds are not met, the balancing circuits stop working.

The working condition for the upper layer circuitsis $V(M)_{\max }-V(M)_{\min }>10 \mathrm{mV}, V(M) s$ are the modules terminal voltages. In each battery module, the working condition for the bottom layer circuitsis $V(B)_{\max }-V(B)_{\min }>5 \mathrm{mV}, V(B) s$ are the terminal voltages of cells in the same module. 
The equalization thresholds could be adjusted according to the system requirements and the accuracy of sampling circuits [1].

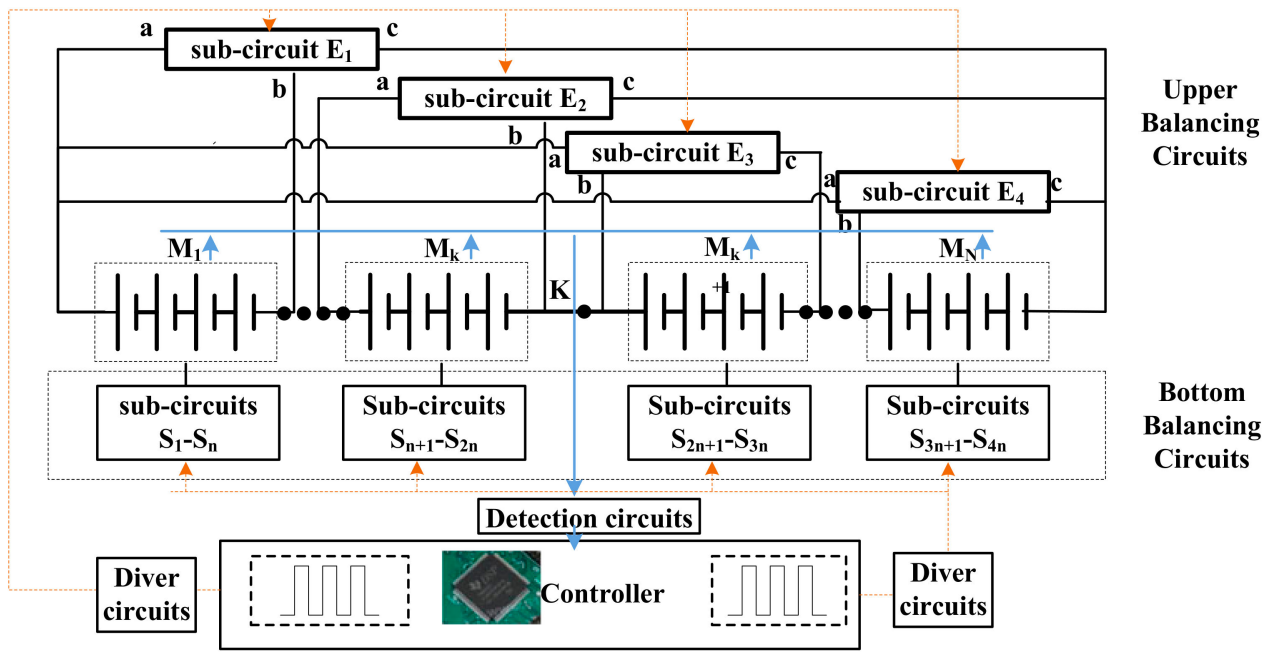

(a)

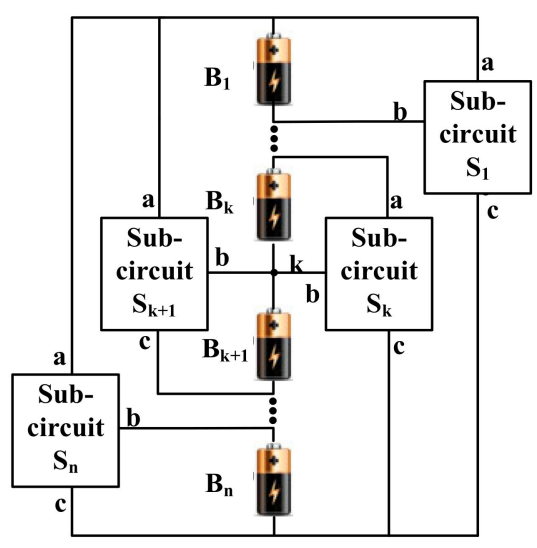

(b)

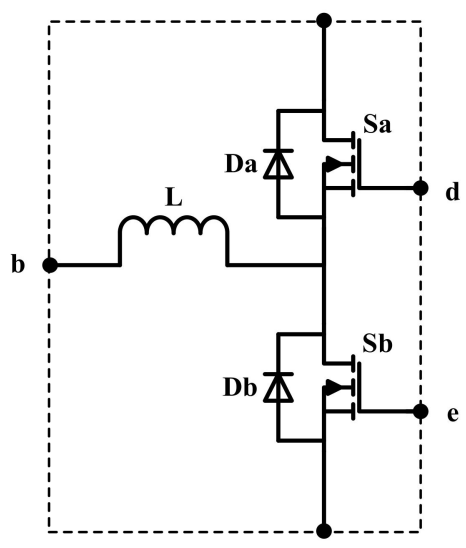

(c)

Figure 2. System configuration of the proposed equalizer (a) structure of the proposed equalizer; (b) schematic diagram of bottom balancing circuit; and (c) schematic diagram of balancing sub-circuit.

\subsection{Equalization Principle}

\subsubsection{The Equalization Principle of the Bottom Layer Circuits}

We use four battery cells connected in series as an example to elaborate the equalization principle of the bottom layer circuits. Firstly, we identify the highest and lowest cell voltage; secondly, we control the corresponding sub-circuit to transfer energy from the cell with the highest voltage to the others in the same module and from the other cells to the one with the lowest voltage synchronously. Suppose that the cell $B_{1}$ is with the highest voltage and cell $B_{4}$ has the lowest voltage in the module $M_{1}$. The equalization principle with two consecutive stages is shown in Figure 3:

Stage 1: charge $L_{1}$ and $L_{4}$.

As shown in Figure $3 a$, the switches $S_{1 a}$ and $S_{4 a}$ are first turned ON. The individual cell $B_{1}$ charges the inductor $L_{1}$; the $B_{1}, B_{2}$ and $B_{3}$ connected in series charge the inductor $L_{4}$. The inductor $L_{1}$ and $L_{4}$ store energy with the current $i_{L_{1}}$ and $i_{L_{4}}$ gradually increasing. Some of the electrical energy is transferred into magnetic energy stored in the inductors. 
Stage 2: discharge $L_{1}$ and $L_{4}$.

As shown in Figure $3 \mathrm{~b}$, the switches $S_{1 a}$ and $S_{4 a}$ are turned OFF. The inductor $L_{1}$ charges cell $B_{2}$, $B_{3}$ and $B_{4}$ through the flywheel diode of $S_{1 b}$; the inductor $L_{4}$ charges cell $B_{4}$ through the flywheel diode of $S_{4 b}$. The most overcharged cell $B_{1}$ and most undercharged cell $B_{4}$ are balanced at the same time. Furthermore, the balancing current does not only flow through $B_{1}$ and $B_{4}$ but also flows through $B_{2}$ and $B_{3}$. This accelerates the balancing process compared to other equalizers.

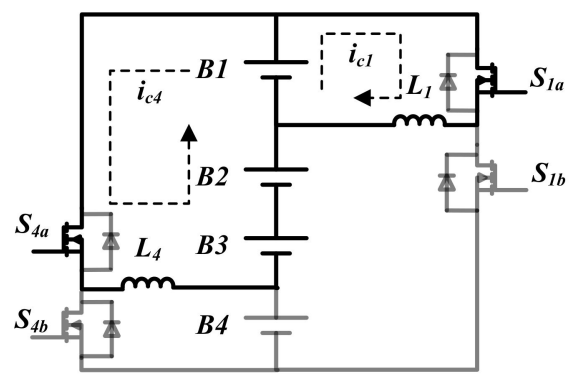

(a)

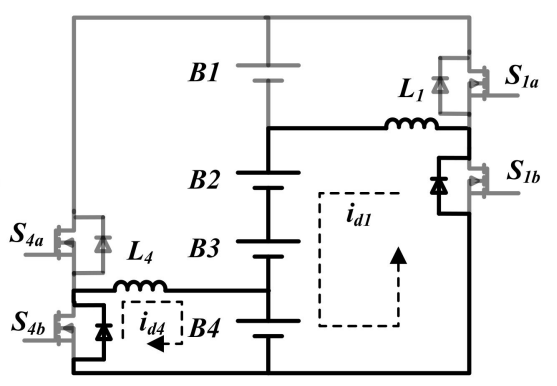

(b)

Figure 3. The equalization principle of proposed equalizer in bottom layer. (a) Charge $L_{1}$ and $L_{4}$ (b) discharge $L_{1}$ and $L_{4}$.

\subsubsection{Equalization Principle of the Upper Layer Balancing Circuits}

Use four battery modules connected in series as an example to elaborate the equalization principle of the upper layer circuits. The module $M_{2}$ is assumed to be overcharged and the module $M_{3}$ is under charged.

As shown in Figure $4 a$, switches $S_{18 a}$ and $S_{19 a}$ are first turned ON. The $M_{2}$ charges the inductor $L_{18} ; M_{1}$ and $M_{2}$ charge the inductor $L_{18}$. Then, as is shown in Figure $4 \mathrm{~b}$, the switches $S_{18 a}$ and $S_{19 a}$ are turned OFF. The inductor $L_{18}$ charges the module $M_{3}$ and $M_{4}$ through the flywheel diode of $S_{18 b}$ and the inductor $L_{19}$ charges module $M_{3}$ through the flywheel diode of $S_{19 b}$.

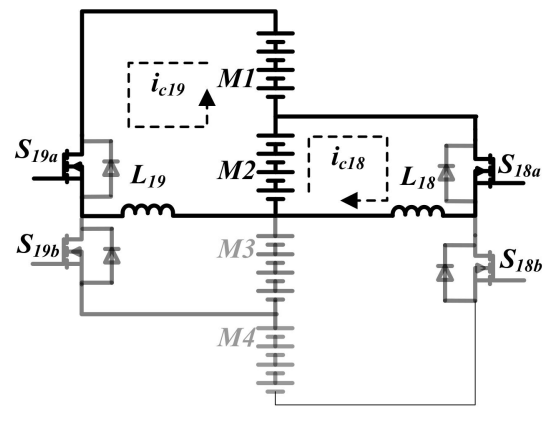

(a)

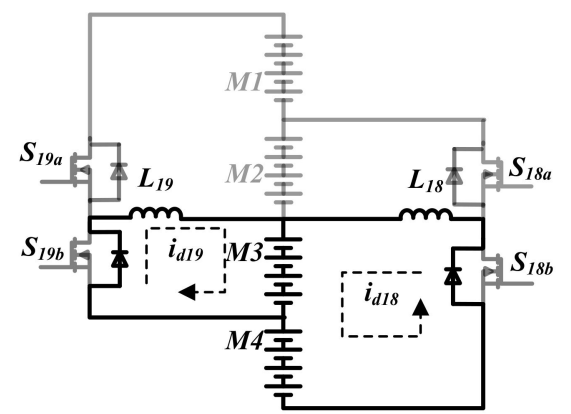

(b)

Figure 4. The equalization principle of proposed equalizer in upper layer. (a) Charge $L_{18}$ and $L_{19}$ (b) discharge $L_{18}$ and $L_{19}$.

\section{Modeling, Analysis and Calculation of the Key Parameters}

In this section several key parameters are analyzed including the balancing current, the inductors current, duty cycle of switch waveform, the switching frequency and the inductances.

\subsection{Modeling of Inductor Current and Duty Cycle of Driving Waveform}

DC-DC (direct current) converters have two working modes: continue current mode (CCM) and discontinuous current mode (DCM), shown in Figure 5. The CCM has a large inductor current with 
feedback control [15]. Considering the magnetic saturation and the complexity of control, DC-DC converters work in the DCM in this paper. In DCM, the inductors can release all absorbed energy in the off-time of the switches and the converters is without current sensors and feedback control [20].

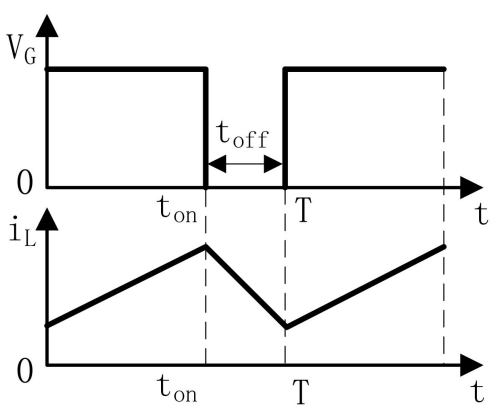

(a)

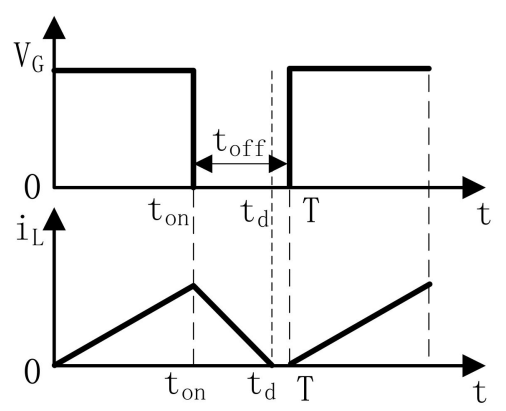

(b)

Figure 5. Two operating mode of DC-DC converter (a) Continuous current mode (CCM) (b) discontinuous current mode (DCM).

During the energy transfer process of the inductor $L_{i}$, denote the voltage across $L_{i}$ by $V_{c}$ when the switch is turned $\mathrm{ON}$, namely the total cells voltage charging $L_{i}$; denote the voltage across $L_{i}$ by $V_{d}$ when the switch is turned OFF, namely the total cells voltage discharging by $L_{i}$ :

$$
\begin{gathered}
V_{c}=R_{o n} i_{L_{i}}+L \frac{d i_{L_{i}}}{d t} \quad 0<t \leq t_{o n} \\
V_{d}=R_{o f f} i_{L_{i}}+L \frac{d i_{L_{i n}}}{d t}-V_{D} \quad t_{o n}<t \leq t_{d}
\end{gathered}
$$

In which, $i_{L_{i}}$ is the inductor current, $V_{D}$ is the forward voltage across the body diode of MOSFET, $R_{o n}$ is the loop total resistance when the switch is turned ON, $R_{\text {off }}$ is the loop total resistance when the switch is turned OFF, $t_{d}$ is the moment that inductor current falls to zero.

This is a first order full response circuit, and the inductor current response is:

$$
\begin{gathered}
i_{L i}=\frac{V_{c}}{R_{o n}}-\frac{V_{c}}{R_{o n}} e^{-t \frac{R_{o n}}{L}}=\frac{V_{c}}{R_{o n}}\left(1-e^{-t \frac{R_{o n}}{L}}\right), 0<t \leq t_{o n} \\
i_{L i}=i_{p} e^{-\left(t-t_{o n}\right)} \frac{R_{o f f}}{L}-\frac{V_{d}+V_{D}}{R_{o f f}}\left(1-e^{-\left(t-t_{o n}\right) \frac{R_{o f f}}{L}}\right), t_{o n}<t \leq t_{d} \\
i_{p}=\frac{V_{c}}{R_{o n}}\left(1-e^{-t_{o n} \frac{R_{o n}}{L}}\right), t=t_{o n}
\end{gathered}
$$

In (5), $i_{p}$ is the peak value of inductor current $i_{L_{i}}$. The resistances $R_{o n}, R_{o f f}$ and $V_{D}$ are ignored since their values are very small. Then the inductor current increases linearly when the switches are turned ON; the inductor current decreases linearly, when the switches are turned OFF:

$$
\begin{gathered}
i_{L_{i}}= \begin{cases}\frac{V_{c}}{L_{i}} t, & 0 \leq t<t_{o n} \\
\frac{V_{c}}{L_{i}} t_{o n}-\frac{V_{d}}{L_{i}}\left(t-t_{o n}\right), & t_{o n} \leq t<t_{d} \\
0, & t_{d} \leq t \leq T\end{cases} \\
i_{p}=\frac{V_{c} \cdot t_{o n}}{L}=\frac{V_{c} \cdot D \cdot T}{L}
\end{gathered}
$$

In (7), $D$ represents duty cycle, and $T$ represents switching period. To make the DC-DC converters working in the DCM, the inductor current need drop to 0 in the off-time of switch. The conditions that the duty cycle need satisfy are derived as follows: $i_{L_{i}}=0$ when $t=t_{d}$; since $T>t_{d}$, when $t=T$ : 


$$
\frac{V_{c}}{L} t_{o n}-\frac{V_{d}}{L}\left(T-t_{\text {on }}\right)<0
$$

Furthermore:

$$
\mathrm{D}<\frac{V_{d}}{V_{c}+V_{d}}=\frac{1}{1+\frac{V_{c}}{V_{d}}}
$$

\subsection{Inductance, Switching Frequency and Equalization Time}

The cell-balancing time is a key indicator for equalizer need to be considered. This problem can not only be solved by improving the equalizer topologies but also by increasing the balancing current. This is because the amount of charge transferred from one cell to others in unit time is proportional to the average value of current through the cell [8]:

$$
\Delta Q=i_{B_{\text {iavr }}} \cdot T \cdot f
$$

In which, $\Delta Q$ is the energy transferred from one cell to the others in one second, $i_{\text {Biavr }}$ is the average current through $B_{i}, T$ is the switching period and $f$ is the switching frequency. In equalization process, the current in balancing circuit is derived as follows:

The peak value of inductor current:

$$
i_{p}=\frac{V_{c}}{L} D T=\frac{V_{c} D}{L f}
$$

The average value of inductor current:

$$
i_{L_{i a v r}}=\frac{1}{2} \frac{V_{c} D}{L f}
$$

In the balancing sub-circuit $S_{i}$, the average value of the cell $B_{i}$ :

$$
i_{B_{\text {iavr }}}=\left\{\begin{aligned}
\frac{1}{2} \frac{V_{c} D^{2}}{L f}, & \text { when the } B_{i} \text { charges } L_{i} \\
\frac{1}{2} \frac{V_{c} D(1-D)}{L f}, & \text { when the } L_{i} \text { charges } B_{i}
\end{aligned}\right.
$$

The average value of inductors current is proportional to the product of $D^{2}$ and $V_{c}$ or $D(1-D)$ and $V_{c}$; is inversely proportional to the product of $L$ and $f$. The values of $D$ and $V_{c}$ cannot adjust at will. The balancing time is inversely proportional to the average value of balancing current, shown in Equation (9), therefore, the balancing time of IBLBE is proportional to the product of $L$ and $f$. In that case, the selection of $L$ and $f$ can achieve different levels of the balancing current and balancing time. Increase the switching frequency $f$ can reduce the volume of inductors and improve equalizer integration. The impact of equalizer topology and the initial distribution sequence of the cells charge will be further discussed in next section.

\section{Simulation}

The simulation model of the proposed equalizer is built in PSIM9.0. In order to reduce the balancing time, $10 \mathrm{~F}$ capacitors are employed to substitute battery cells $[8,14]$ with initial voltage shown in Table 1. The switching frequency is set as $10 \mathrm{kHz}$. Cell balancing based on voltage inconsistency is more easily implemented and more common $[15,20,22]$. In this paper, the cells terminal voltages are employed as the index of inconsistency.

It is assumed that the capacitors, inductors and switches are all lumped element. Moreover, the influence of parasitic inductance and parasitic capacitance and the deviation generated by AD (anolog to digital) transfer are ignored. 
Table 1. Initial voltages of 16 cells.

\begin{tabular}{ccccccccc}
\hline Modules & \multicolumn{2}{c}{$M_{\mathbf{1}}$} & \multicolumn{2}{c}{$\boldsymbol{M}_{\mathbf{2}}$} & \multicolumn{2}{c}{$\boldsymbol{M}_{\mathbf{3}}$} & \multicolumn{2}{c}{$\boldsymbol{M}_{\mathbf{4}}$} \\
\hline Voltage (V) & \multicolumn{2}{c}{15.646} & \multicolumn{2}{c}{15.617} & \multicolumn{2}{c}{15.676} & \multicolumn{2}{c}{15.587} \\
Cells & $B_{1}$ & $B_{2}$ & $B_{3}$ & $B_{4}$ & $B_{5}$ & $B_{6}$ & $B_{7}$ & $B_{8}$ \\
Voltage (V) & 3.961 & 3.935 & 3.909 & 3.871 & 3.955 & 3.897 & 3.930 & 3.864 \\
SOC (\%) & 65 & 61 & 57 & 53 & 64 & 56 & 60 & 52 \\
Cells & $B_{9}$ & $B_{10}$ & $B_{11}$ & $B_{12}$ & $B_{13}$ & $B_{14}$ & $B_{15}$ & $B_{16}$ \\
Voltage (V) & 3.922 & 3.858 & 3.948 & 3.889 & 3.852 & 3.942 & 3.916 & 3.877 \\
SOC (\%) & 59 & 51 & 63 & 55 & 50 & 62 & 58 & 54 \\
\hline
\end{tabular}

SOC (\%): SOC values corresponding to each cell voltage are for a 2.6 Ah Sanyo ternary lithium battery. $V_{B \max }=3.961 \mathrm{~V}, V_{B \min }=3.852 \mathrm{~V}$, maximum voltage gap is $109 \mathrm{mV}$, maximum SOC gap is $15 \%$. $V_{M \max }=15.676 \mathrm{~V}, V_{M \min }=15.587 \mathrm{~V}$, maximum voltage gap is $89 \mathrm{mV}$.

\subsection{Equalization Simulation for IBLBE and PAE}

\subsubsection{Equalization Simulation for IBLBE and PAE with 4 Cells}

The circuit diagrams and the equalization flow charts of the two equalizers are shown in Figures 6 and 7. The balancing time of the two topologies both depend strongly on the initial distribution sequence of the cells' charge. In fact, some charge transferred from one cell to another occurs directly; in other cases, the charge transferred from one cell to another only occurs after multiple transfers between groups of cells. Therefore, the balancing time depends on the initial distribution sequence of the cell charges.

A statistic work has been done to obtain the average balancing time in different initial distribution sequence of the cell charges for PAE and IBLBE. Take the four cells battery string as an example. The number of different distribution sequence is $\mathrm{A}_{4}^{4}=24$, and only 12 are independent samples. The initial voltages of the four cells are A $=3.961 \mathrm{~V}, \mathrm{~B}=3.935 \mathrm{~V}, \mathrm{C}=3.909 \mathrm{~V}, \mathrm{D}=3.871 \mathrm{~V}$, the values of SOC are $65 \%, 61 \%, 57 \%, 53 \%$ for a 2.6 Ah Sanyo ternary lithium battery. Set the equalization threshold as $3 \mathrm{mv}$ for the two equalizers. Table 2 shows the 12 independent samples and their balancing time in PAE and IBLBE. Table 3 shows the comparison between PAE and IBLBE in 4 cells battery string. The proposed equalizer IBLBE uses two more MOSFETs and one more inductor than PAE. In the 12 independent samples, there are nine samples in which the balancing time of IBLBE is shorter than PAE. The IBLBE reduces balancing time by $29 \%$ in average. The balancing time variance of IBLBE is much smaller than that of PAE, which means the IBLBE has a relatively stable balancing time than PAE in different initial distribution sequence of the cell charges. In PAE, the balancing time is usually longer when the voltage difference in the first layer equalizer becomes larger, such as ADBC, ADCB, BCAD, DABC.

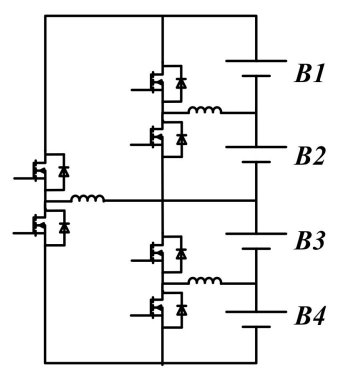

(a)

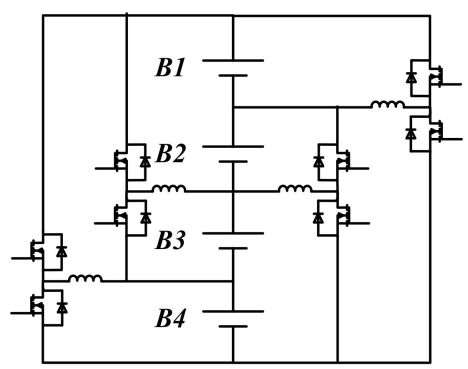

(b)

Figure 6. Circuit diagrams of the PAE and the IBLBE with 4 cells. (a)The PAE; (b) the IBLBE. 


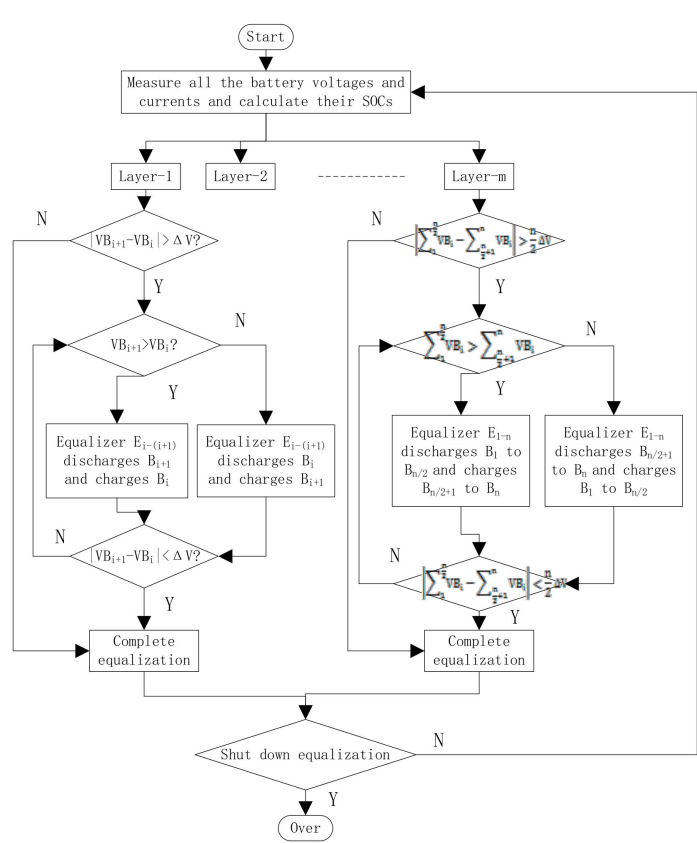

(a)

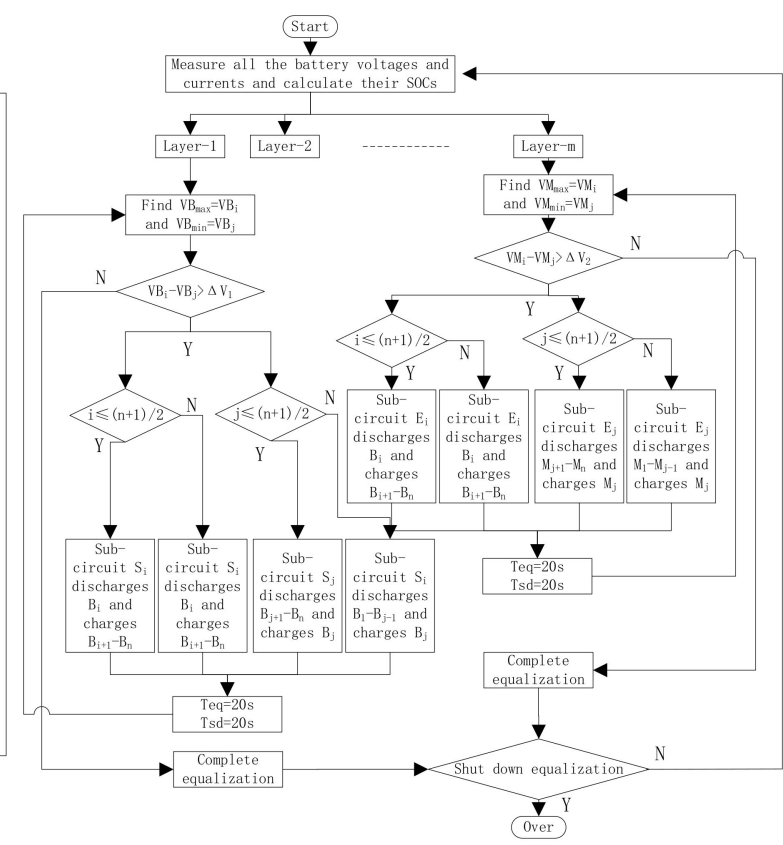

(b)

Figure 7. The equalization flow charts of the PAE and the IBLBE. (a) The PAE; (b) the IBLBE.

Table 2. The balancing time in different distribution sequence for PAE and IBLBE (time unit: second).

\begin{tabular}{ccccccccccccc}
\hline & ABCD & ABDC & ACBD & ACDB & ADBC & ADCB & BACD & BADC & BCAD & BDAC & CABD & DABC \\
\hline PAE & 0.4035 & 0.4035 & 0.6978 & 0.6978 & 0.993 & 0.993 & 0.4035 & 0.4035 & 0.993 & 0.6978 & 0.6978 & 0.993 \\
IBLBE & 0.3315 & 0.5721 & 0.3933 & 0.7016 & 0.6757 & 0.6984 & 0.2826 & 0.5264 & 0.4016 & 0.6907 & 0.3405 & 0.3262 \\
\hline
\end{tabular}

Table 3. The comparison between PAE and IBLBE in 4 cells battery string (G: Good; M: Marginal; P: Poor).

\begin{tabular}{cccccccc}
\hline & $\begin{array}{c}\text { Expected } \\
\text { Time (s) }\end{array}$ & Variance & $\begin{array}{c}\text { Cell } \\
\text { Number }\end{array}$ & MOSFET & Inductor & $\begin{array}{c}\text { Control } \\
\text { Complexity }\end{array}$ & $\begin{array}{c}\text { Algorithm } \\
\text { Variety }\end{array}$ \\
\hline PAE & 0.6981 & 0.05792 & 4 & 6 & 3 & $\mathrm{G}$ & $\mathrm{M}$ \\
IBLBE & 0.4950 & 0.02543 & 4 & 8 & 4 & $\mathrm{G}$ & $\mathrm{G}$ \\
\hline
\end{tabular}

Furthermore, the IBLBE can use more different algorithms than PAE since all the balancing sub-circuits in IBLBE are independent.

\subsubsection{Equalization Simulation for IBLBE and PAE with 16 Cells}

The initial voltages of cells and modules are shown in Table 1. We built layered balancing circuits for IBLBE and PAE to compare the balancing speed between IBLEB and PAE with a 16 cell battery string. The 16 cell battery string is divided into four battery modules, and each module has four cells connected in series.

Figure 8 presents the cell voltages trajectories in PAE and IBLBE. As is shown in Figure 8 , the IBLBE stops equalization at about $0.7125 \mathrm{~s}$, and the PAE stops at $0.9969 \mathrm{~s}$. The equalization between four battery modules is much faster than the equalization in the bottom layer (or the first layer).

In Figure 8a, the slowest battery module is M4, and its balancing time is $0.9969 \mathrm{~s}$ which puts off the whole PAE equalization process. In Figure $8 b$, the slowest battery module is $M_{3}$, and its balancing time is $0.7125 \mathrm{~s}$, which equals the whole balancing time of IBLBE. In module $M_{1}$, the balancing speed of IBLBE is faster than PAE. In module $M_{2}$ and $M_{3}$, the balancing time is the same in PAE. The IBLBE is a little bit slower than PAE in $M_{3}$, but it is faster than PAE in $M_{2}$. 

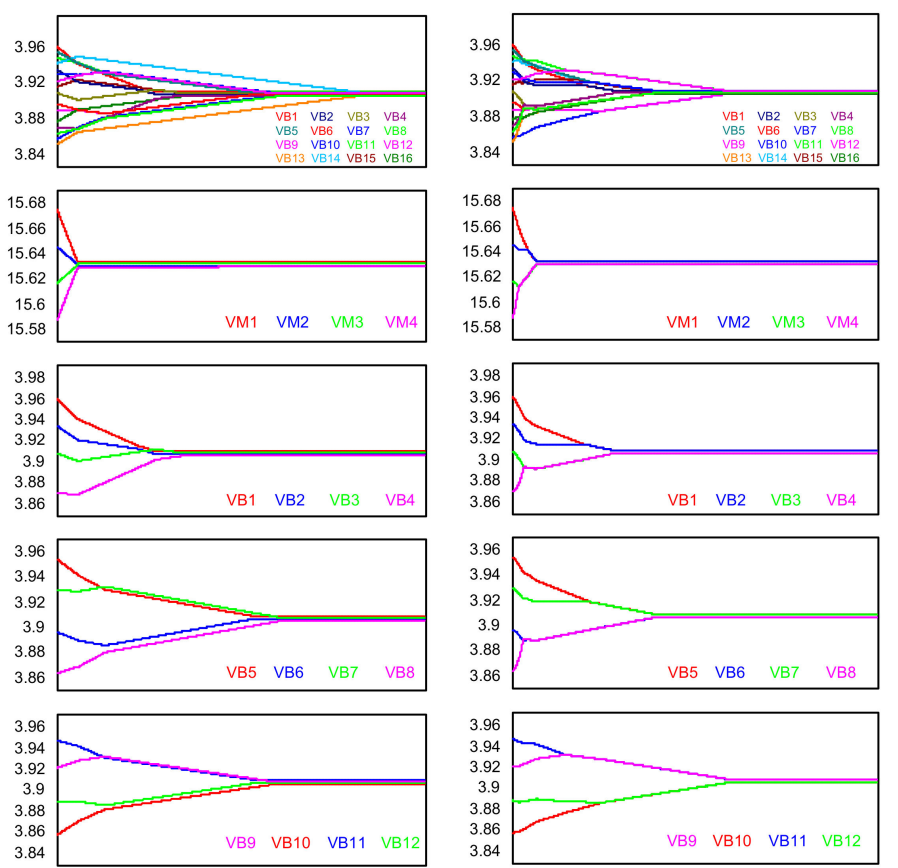

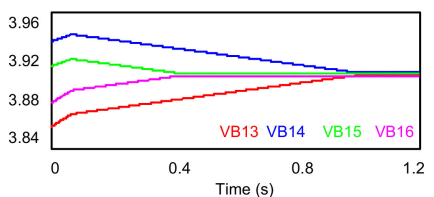

(a)

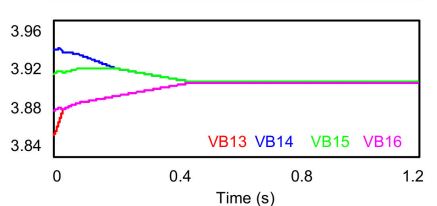

(b)

Figure 8. 16 cells voltage trajectories in simulation (a) PAE (b) IBLBE.

Thus, the balancing time for the whole battery string depends on the slowest battery module. The balancing time for each battery module depends on the initial distribution sequence of cell charges. The IBLBE can achieve faster active equalization than PAE, because the balancing time fluctuation of IBLBE is much smaller than that of PAE in different distribution sequence of cells charge and the expected balancing time of IBLBE is shorter than PAE. For example, the module $M_{4}$ stops equalization at $0.9969 \mathrm{~s}$ in PAE, but it only needs $0.4332 \mathrm{~s}$ in IBLBE.

To quantitatively evaluate the expected balancing time for the two topologies with 16 cells battery string, the statistical model is simplified in this paper. Suppose that the initial charges of cells are the same in different modules, but the distribution sequence is different. Thus, balancing operation only occurs between cells in each module and not occurs between battery modules. The whole balancing time depends on the slowest module. As shown in Table 2, the PAE has three independent samples for four cells; IBLBE has about four "independent" samples for four cells (the samples with similar speeds are sorted into the same independent sample). After probabilistic and statistical computing, the expected balancing time of IBLBE is $0.6531 \mathrm{~s}$, the expected balancing time of PAE is $0.9311 \mathrm{~s}$. IBLBE reduces the expected balancing time by about $30 \%$.

\subsection{Equalization Simulation for Other Topologies with 16 Cells}

Han has done some comparisons between PAE [15], IBAE [18], PAEBMWT [25] and DTSCE [10]. The comparison results showed that the PAE balancing time of PAE is the shortest, and it can be reduced by $50 \%$, compared with IBAE. Furthermore, the PAE has good performance in balancing efficiency, and control complexity. In this part, some further comparison between switched-capacitor-based equalizer (SCBE) [8], chain structure switched-capacitor equalizer (CSSCE) [9], single switched-inductor equalizer (SSIE) [21] and buck-boost-and-LC-resonant based equalizer (BBLCRE) [13] has been presented. The equalization process of the four equalizers is shown in Figure 9. 

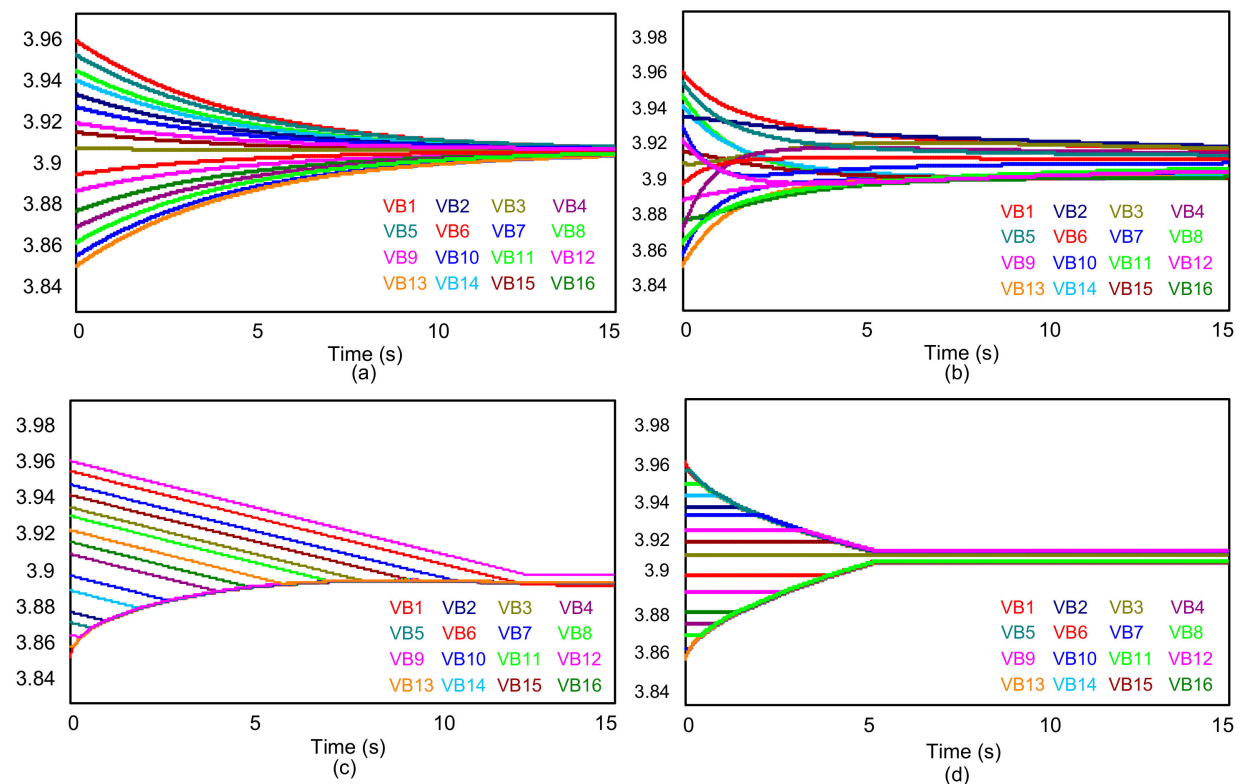

Figure 9. Simulation results of four different equalizers. (a) SCBE, C $=220 \mu \mathrm{F}, f=10 \mathrm{kHz}$ (b) CSSCE, $\mathrm{C}=220 \mu \mathrm{F}, f=10 \mathrm{kHz}$ (c) BBLCRE, Vout-buck $=4.5 \mathrm{~V}, \mathrm{Cr}=100 \mu \mathrm{F}, \mathrm{Lr}=50 \mu \mathrm{H}, \mathrm{R}=0.2 \Omega, f=2230 \mathrm{~Hz}$ (d) SSIE, $\mathrm{L}=100 \mu \mathrm{H}, f=10 \mathrm{kHz}$.

Table 4 presents the comparison between different equalizers. The SCBE proposed by Ye and Cheng, has a simply topology and its balancing speed is independent of both of the number of battery cells and initial distribution sequence of cell voltages. However its balancing speed is much slower than IBLBE. The balancing time of SCBE is 21 times longer than that of IBLB. The CSSCE can't realize accurate equalization between 16 cells or a large number of cells during an acceptable time. The BBLCRE has excellent performance in balancing efficiency, but the balancing speed is slow. The balancing speed of BBLCRE is proportional to the voltages difference across LC resonant converter, but the balancing efficiency is inversely proportional to it. Thus, balancing efficiency and speed, the BBLCRE can't have both. In addition, it needs more relays than other topologies. When the V out of the buck-boost converter is set to $4.5 \mathrm{~V}$, and the ideal efficiency of LC resonant converter is about $86.7 \%$. In this case, the balancing time of BBLCRE is 12 times longer than that of IBLBE. The SSIE uses only one inductor. However, this equalizer has a complex switch matrix which needs to operate four switches in a loop in each switching period and it takes a long time to serve all cells in a long battery string with only one balancing bypass. Furthermore, the balancing time of SSIE is about eight times longer than that of IBLBE.

Table 4. Comparison of different equalizers with 16 cells (Balancing time: the time when the maximum voltage gap in the battery string is lower than $5 \mathrm{mV}$. G: Good; M: Marginal; P: Poor.)

\begin{tabular}{ccccccc}
\hline & IBLBE & PAE & SCBE & CSSCE & BBLCRE & SSIE \\
\hline Balancing time (s) & 0.6531 & 0.9311 & 14.053 & $>>15$ & 12.625 & 5.346 \\
MOSFET & 40 & 30 & 32 & 32 & 5 & 34 \\
Capacitors & 0 & 0 & 16 & 16 & 3 & 0 \\
Inductors & 20 & 15 & 0 & 0 & 2 & 1 \\
Diodes & 0 & 0 & 0 & 0 & 0 & 34 \\
Relays & 0 & 0 & 0 & 0 & 17 & 0 \\
Efficiency & $\mathrm{G} / \mathrm{M}$ & $\mathrm{G} / \mathrm{M}$ & $\mathrm{G}$ & $\mathrm{G}$ & $\mathrm{G} / \mathrm{M}$ & $\mathrm{M}$ \\
Control complexity & $\mathrm{G}$ & $\mathrm{G}$ & $\mathrm{G}$ & $\mathrm{G}$ & $\mathrm{P}$ & $\mathrm{P}$ \\
\hline
\end{tabular}




\section{Experimental Results}

In order to further verify the equalization principles and show the balancing performance of the proposed equalizer, a balancing system is implemented and tested with sixteen 2.6 Ah Sanyo ternary lithium batteries. Table 5 summarizes the parameters of IBLBE. The inductances and the resistances in Table 5 are measured by a TH2810D LCR Meter (Tonghui, Changzhou, China). The initial cell voltages and SOCs are shown in Table 1.

Table 5. Component values used for the equalizer.

\begin{tabular}{ccccc}
\hline Parameters & \multicolumn{3}{c}{ Values } \\
\hline Battery string & Sanyo ternary lithium batteries & $B_{1}-B_{16}$ & $2.6 \mathrm{Ah}$ & - \\
Equalizer & MOSFET & $S_{1 a}, S_{1 b}-S_{20 a}, S_{20 b}$ & IRF530 & $\mathrm{R}_{\mathrm{DS}(\text { on) }}<0.090 \Omega$ \\
- & Inductance & $L_{1}-L_{16}$ & $99.1-101.5 \mu \mathrm{H}$ & $\mathrm{R}<0.0450 \Omega$ \\
- & - & $L_{17}-L_{20}$ & $328.2-332.4 \mu \mathrm{H}$ & $\mathrm{R}<0.0822 \Omega$ \\
\hline
\end{tabular}

In every equalization cycle, the equalization time $t_{e q}=10 \mathrm{~s}$, and the standing time $t_{s d}=10 \mathrm{~s}$. The standing time in every equalization cycle is aimed at eliminating the polarization voltage of cells. Therefore, it is more accurately to test the real open-circuit voltages (OCVs) of cells, and issue the balancing instructions.

Figure 10a shows the experimental waveforms of inductors current $i_{L_{1}}$ and $i_{L_{19}}$ and the duty cycle of PWM is $65 \%$; Figure 10b shows the experimental waveforms of inductors current $i_{L_{4}}$ and $i_{L_{20}}$ and the duty cycle of PWM is $24 \%$. The inductors current is discontinuous and varies in saw-tooth waveform. The peak value of $i_{L_{1}}$ comes to $2.2 \mathrm{~A}$; the peak value of $i_{L_{19}}$ is about $2.1 \mathrm{~A}$; the peak value of $i_{L_{4}}$ is $2.5 \mathrm{~A}$; the peak value of $i_{L_{20}}$ is about $2.2 \mathrm{~A}$. These agree with the theoretical analysis in Section 3.

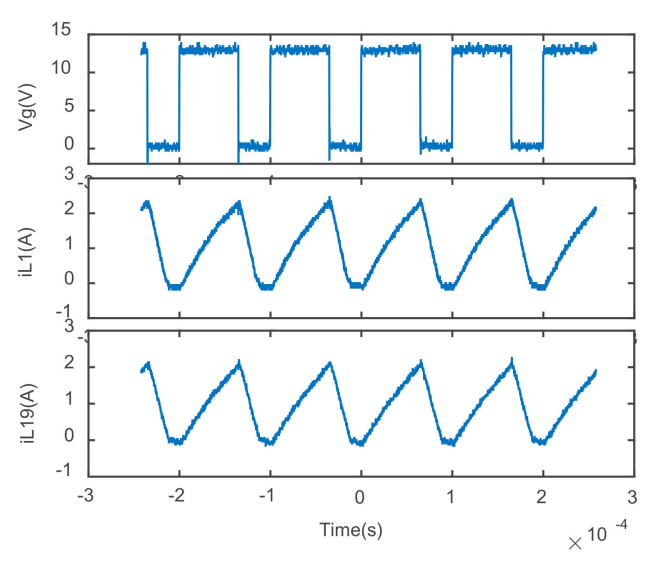

(a)

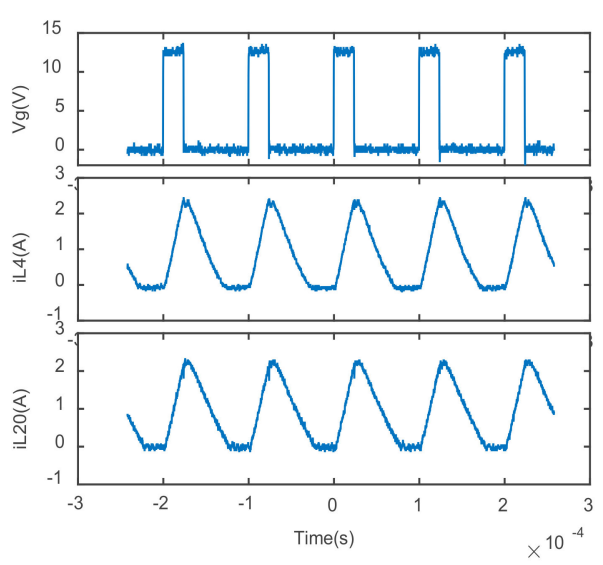

(b)

Figure 10. Experimental waveform in the beginning (a) $i_{L_{1}}$ and $i_{L_{19}}(\mathbf{b}) i_{L_{14}}$ and $i_{L_{20}}$.

Figure 11 shows the 16 cells' voltage trajectories in the equalization process. Figure 12 presents the 16 cells' voltage before and after equalization. The initial voltage gap shown in Table 1 is $103 \mathrm{mV}$, and it decreases to $8 \mathrm{mV}$ at about $37 \mathrm{~min}$.

The initial SOC gap is $15 \%$, and the final SOC gap decreases to $1.2 \%$. The accuracy of the equalization is excellent. The balancing time in Figure 11 agrees with the simulation result in Figure $8 \mathrm{~b}$ after an equivalent calculation method. 


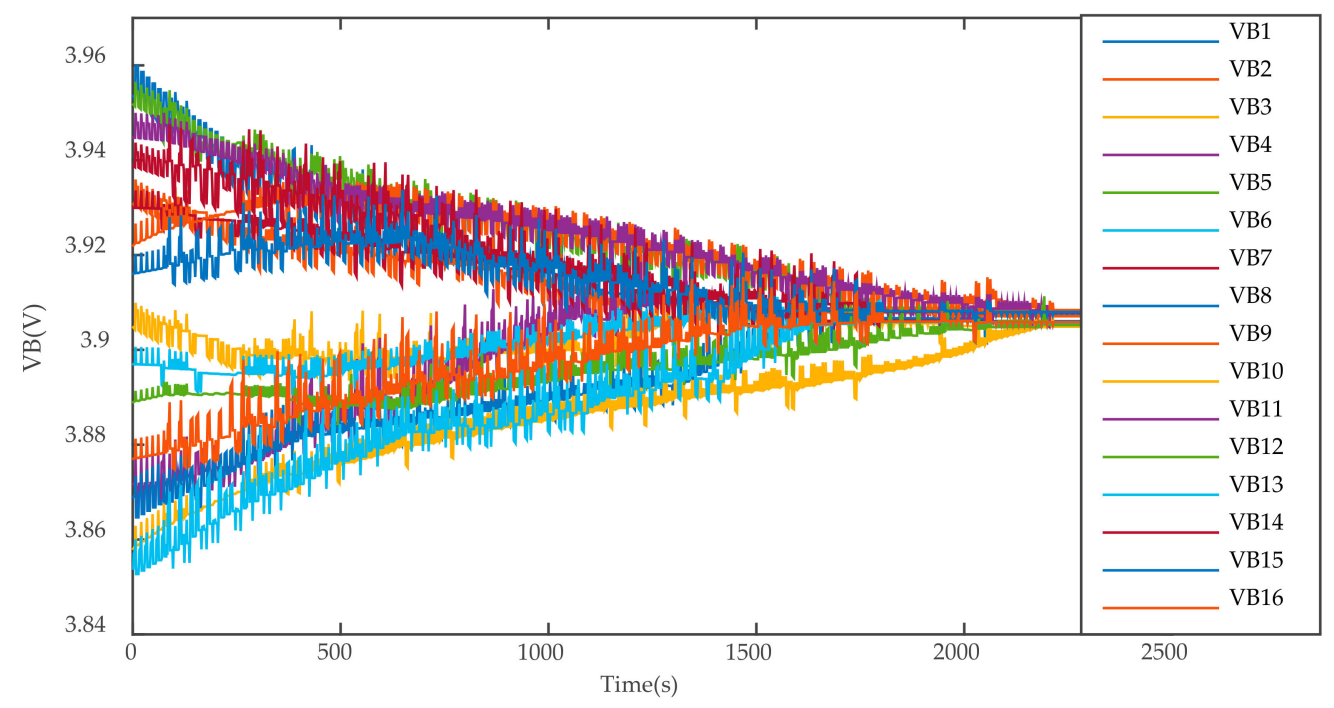

Figure 11. Voltage trajectories of 16 cells in the equalization process.

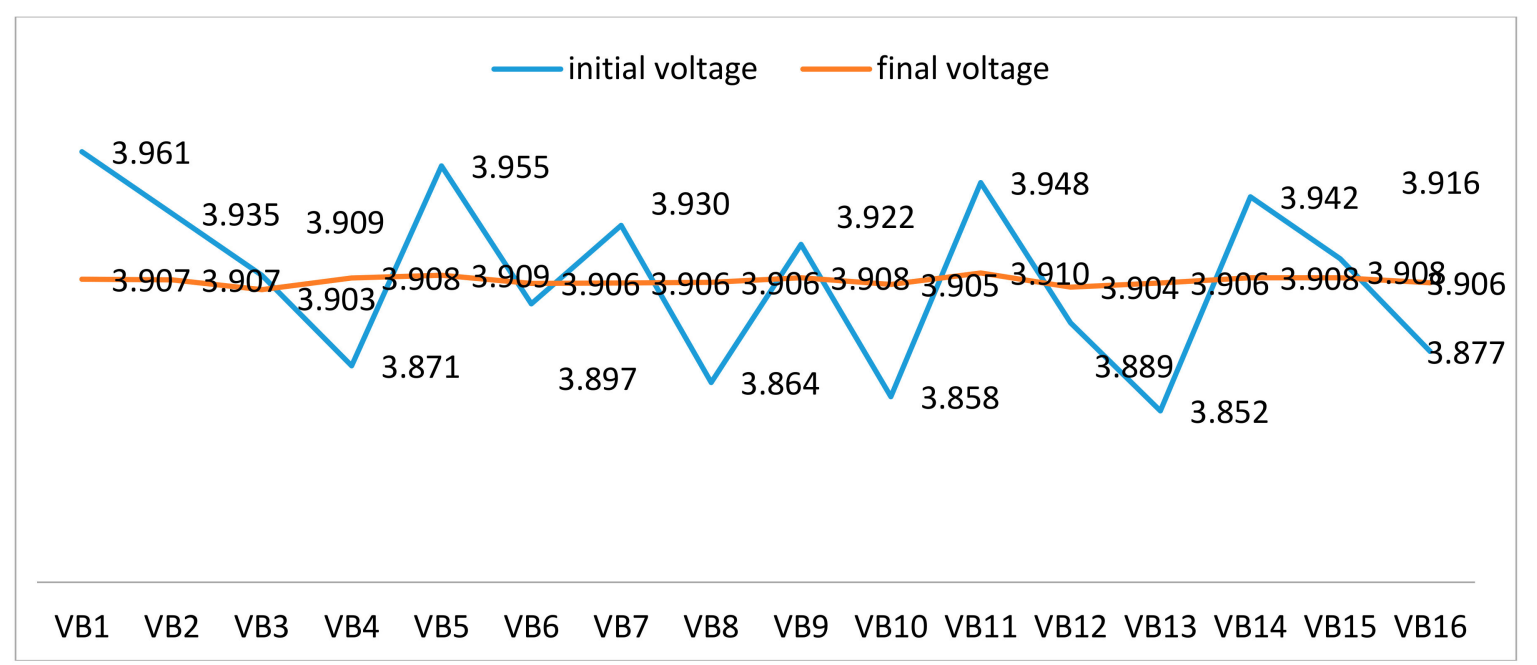

Figure 12. Voltage of16 cells before and after equalization.

\section{Conclusions}

In this paper, a novel inductor-based layered bidirectional equalizer (IBLBE) for series connected battery strings is proposed. The equalization principle, modeling of balancing current and duty cycle, simulations for different equalizer with four and 16 cells and experiments for IBLBE with 16 cells are presented. The proposed equalizer has the characteristics of multiple balancing path, easy control and higher exploitability in equalization strategy. Adjusting the product $f^{*} L$ of switching frequency and inductance can achieve the different levels of balancing current and balancing speed. Comparison between different equalizers demonstrates the proposed equalizer has a higher level balancing speed than other equalizers based on switched-capacitor or switched-inductor converters. A statistic study on the balancing speed shows that the IBLBE reduces the balancing time by $30 \%$ in average, compared to PAE. Simulation and experimental results indicate that the IBLBE has a good performance in balancing speed and balancing accuracy, and it is more suitable for a large number of cells. As future work, some new equalization strategies and algorithms reported in [26,27], will be developed for IBLBE. Modeling balancing efficiency and balancing time reported in [4], will be applied to IBLBE. 
Acknowledgments: This work is supported by the DongGuan Innovative Research Team Program (No. 2014607119) and the Guangdong Province Science and Technology Program (No. 2014B010125002). I would like to express my deepest gratitude to my supervisor, Longyun Kang, who has provided me with valuable guidance at every stage of this paper-writing. I would also like to thank the anonymous reviewers for dedicating the time to review my paper despite their busy schedules.

Author Contributions: This research article has five authors. The circuit structure was designed by Shubiao Wang and Longyun Kang. Shubiao Wang, Longyun Kang, XiangweiGuo and Ming Liu conceived the research methods and control strategies. Shubiao Wang and Zefeng Wang designed and performed the experiments. Longyun Kang contributed the experimental environment. Shubiao Wang wrote the paper.

Conflicts of Interest: The authors declare no conflict of interest.

\section{References}

1. Tan, X. Battery Management Systems on Power Batteries: Applied Technology and Advanced Theories; Zhongshan University Press: Guangzhou, China, 2014; pp. 79-108.

2. Guo, X. Study on State of Charge Estimation and Equalization Technique of Electric Vehicle Battery. Ph.D. Thesis, South China University of Technology (SCUT), Guangzhou, China, 2016.

3. Li, Y.; Han, Y. A module-integrated distributed battery energy storage and management system. IEEE Trans. Power Electron. 2016, 31, 8260-8270. [CrossRef]

4. Baronti, F.; Roncella, R.; Saletti, R. Performance comparison of active balancing techniques for lithium-ion batteries. J. Power Sources 2014, 267, 603-609. [CrossRef]

5. Lozano, J.G.; Cadaval, E.R. Battery equalization active methods. J. Power Sources 2014, 246, 934-949. [CrossRef]

6. Shang, Y.; Zhang, C.; Cui, N.; Guerrero, J.M. A cell-to-cell battery equalizer with zero-current switching and zero-voltage gap based on quasi-resonant LC converter and boost converter. IEEE Trans. Power Electron. 2015, 30, 3731-3747. [CrossRef]

7. Daowd, M.; Omar, N.; Bossche, P.V.D.; Mierlo, J.V. A review of passive and active battery balancing based on Matlab/Simulink. Int. Rev. Electr. Eng. 2011, 6, 2974-2989.

8. Ye, Y.; Cheng, K.W.E.; Fong, Y.C.; Xue, X.; Lin, J. Topology modelling and design of switched-capacitor-based cell balancing systems and their balancing exploration. IEEE Trans. Power Electron. 2017, 32, 4444-4454. [CrossRef]

9. Kim, M.; Kim, C.; Kim, J.; Moon, G. A chain structure of switched capacitor for improved cell balancing speed of lithium-ion batteries. IEEE Trans. Ind. Electron. 2014, 61, 3989-3999. [CrossRef]

10. Baughman, A.C.; Ferdowsi, M. Double-tiered switched-capacitor battery charge equalization technique. IEEE Trans. Ind. Electron. 2008, 55, 2277-2285. [CrossRef]

11. Ye, Y.; Cheng, K.W.E. An automatic switched-capacitor cell balancing circuit for series-connected battery string. Energies 2016, 9, 138. [CrossRef]

12. Lee, Y.S.; Cheng, G.T. Quasi-resonant zero-current-switching bidirectional converter for battery equalization applications. IEEE Trans. Power Electron. 2006, 21, 1213-1224. [CrossRef]

13. Li, Z.; Shang, Y.; Bin, D.; Zhang, C. A pack-to-cell-to-pack battery equalizer with soft-switching based on buck-boost and bidirectional LC resonant converters. In Proceedings of the 2016 IEEE Energy Conversion Congress and Exposition, Milwaukee, WI, USA, 18-22 September 2016.

14. Ye, Y.; Cheng, K.W.E.; Yeung, Y.P.B. Zero-Current Switching Switched-Capacitor Zero-Voltage-Gap Automatic Equalization System for Series Battery String. IEEE Trans. Power Electron. 2012, 27, 3234-3242.

15. Dong, B.; Li, Y.; Han, Y. Parallel architecture for battery charge equalization. IEEE Trans. Power Electron. 2015, 30, 4906-4913. [CrossRef]

16. Uno, M.; Kukita, A. Current sensor less equalization strategy for single-switch voltage equalizer using multi-stacked buck-boost converters for photovoltaic modules under partial shading. IEEE Trans. Ind. Appl. 2017, 53, 420-429. [CrossRef]

17. Uno, M.; Tanaka, K. Single-switch multi-output charger using voltage multiplier for series-connected lithium-ion battery/super-capacitor equalization. IEEE Trans. Ind. Electron. 2013, 60, 3227-3239. [CrossRef]

18. Phung, T.H.; Collet, A.; Crebier, J.C. An optimized topology for next-to-next balancing of series-connected lithium-ion cells. IEEE Trans. Power Electron. 2014, 29, 4603-4613. [CrossRef]

19. Guo, X.; Kang, L.; Huang, Z.; Yao, Y.; Yang, H. Research on a novel power inductor-based bidirectional lossless equalization circuit for series-connected battery packs. Energies 2015, 8, 5555-5576. [CrossRef] 
20. Li, W.; Liao, X.; Gao, Z. A modular equalizer using buck/boost converters with Snubber capacitors for series-connected supercapacitors. In Proceedings of the IEEE International Conference on Industrial Technology (ICIT), Taipei, Taiwan, 14-17 March 2016; pp. 1904-1909.

21. Yarlagadda, S.; Hartley, T.T.; Husain, I.A. Battery management system using an active charge equalization technique based on a DC/DC converter topology. In Proceedings of the IEEE Energy Conversion Congress and Exposition (ECCE), Phoenix, AZ, USA, 17-22 September 2011; pp. 1188-1195.

22. Zhou, Z.; Shang, Y.; Duan, B.; Zhang, C. An any-cell(s)-to-any-cell(s) equalizer based on bidirectional inductor converters for series connected battery string. In Proceedings of the IEEE Conference on Industrial Electronics and Applications, Hefei, China, 5-7 June 2016; pp. 2511-2515.

23. Chen, Y.; Liu, X.; Cui, Y.; Zou, J.; Yang, S. A multi-winding transformer cell-to-cell active equalization method for lithium-ion batteries with reduced number of driving circuits. IEEE Trans. Power Electron. 2016, 31, 4916-4929. [CrossRef]

24. Li, S.; Mi, C.C.; Zhang, M. A high-efficiency active battery-balancing circuit using multi-winding transformer. IEEE Trans. Ind. Appl. 2013, 49, 198-207. [CrossRef]

25. Kutkut, N.H. Non-dissipative current diverter using a centralized multi-winding transformer. In Proceedings of the 28th IEEE Power Electronics Specialists Conference, Saint Louis, MO, USA, 22-27 June 1997; pp. 648-654.

26. Lee, Y.S.; Cheng, M. Intelligent control battery equalization for series connected lithium-ion battery strings. IEEE Trans. Ind. Electron. 2005, 52, 1297-1307. [CrossRef]

27. Li, W.; Kang, L.; Guo, X.; Yao, Y. Multi-objective predictive balancing control of battery packs based on predictive current. Energies 2016, 9, 298. [CrossRef]

(C) 2017 by the authors. Licensee MDPI, Basel, Switzerland. This article is an open access article distributed under the terms and conditions of the Creative Commons Attribution (CC BY) license (http:/ / creativecommons.org/licenses/by/4.0/). 